\title{
Identité du créateur de start-up et accompagnement de l'entrepreneur technologique
}

\section{The Identity of Start-up Founder and Hi-tech Entrepreneur Coaching}

\author{
Gérard A. Kokou Dokou ${ }^{1}$ \\ ${ }^{1}$ Recherche Interdisciplinaire en Management et Economie Lab (RIME-Lab), EA 7396, \\ Université du Littoral Côte d'Opale
}

\begin{abstract}
RÉSUMÉ. Une start-up est une société innovante avec un fort ancrage au niveau des nouvelles technologies et de fortes perspectives de marché. L'entrepreneur de ce type d'organisation est souvent centré sur son cœur de métier par l'exploitation de ses expériences, connaissances et compétences. Les besoins d'accompagnement sont importants d'autant que des compétences complémentaires sont nécessaires au démarrage et au décollage de l'affaire de nature complexe. En ce sens, les logiques d'action retenues par le créateur sont tellement impactées par ses besoins d'être, de savoir et de pouvoir au point de reconstituer le substrat de son identité entrepreneuriale. Ces trois besoins, que l'accompagnant doit inscrire dans sa démarche d'expert-conseil, sous-tendent le pouvoir d'actions du créateur au sens des théories des ressources et de l'effectuation. Pour vérifier un tel constat, une étude qualitative est conduite sous forme d'entretien collectif (focus group) réunissant 8 créateurs de start-up. Les discussions ont été enregistrées et retranscrites intégralement. Le corpus ainsi obtenu est passé au crible des méthodes d'analyse sémantiques et structurales. L'approche interprétativiste conduit à faire valoir l'importance des composantes de l'identité entrepreneuriale du créateur d'une start-up. De telles composantes identitaires prennent la forme de leviers d'action pour l'expert-conseil de l'entrepreneur technologique.

ABSTRACT. A start-up is an innovating company deeply rooted in technologies and with high market prospects. In such structure, the entrepreneur is often focused on his core business, making use of his experiences, knowledge and skills. Support is all the more needed as further skills are necessary to start and to take off in a complex business environment. The founder's operating logics are thus so deeply affected by his needs to be, to know and to be able to that they recreate the essence of his entrepreneurial identity. These three requirements, which the coach must all include in his advising strategy, underpin the founder's empowerment according to resource and application theories. In order to test this observation, a qualitative survey has been carried out in the form of group interview (focus group), bringing together 8 start-up founders. The debates have been recorded and transcribed in their entirety. The achieved corpus is then meticulously studied through semantic and structural analytical methods. The interpretative approach leads to asserting the extent of the components of entrepreneurial identity of the start-up founder. Such identity components turn out to be instruments for the coach of technology entrepreneur.

MOTS-CLÉS. entrepreneur technologique, start-up, technologie numérique, identité entrepreneuriale, accompagnement KEYWORDS. technology entrepreneur, start-up, digital technology, entrepreneurial identity, coaching
\end{abstract}

\section{Introduction}

Les besoins d'accompagnement du créateur de start-up sont nombreux. En effet, une start-up est une société innovante avec un fort ancrage au niveau des nouvelles technologies et de fortes perspectives de marché. L'entrepreneur est souvent centré sur son cœur de métier par l'exploitation de ses expériences, connaissances et compétences. Il a alors du mal à savoir composer avec les parties prenantes qui ont des attentes plurielles et diverses. Les besoins d'accompagnement sont importants d'autant que des compétences complémentaires sont nécessaires au démarrage et au décollage de l'affaire de nature complexe. En ce sens, l'accompagnement peut intégrer cinq niveaux d'interventions (Castrogiovanni, 1996 ; Ries, 2011 ; Eastman et al., 2014) : l'expertise (formulation des solutions), le coaching (aide à la recherche des solutions), la pédagogie (transmission de connaissances et de savoir-faire), le financement (aide à l'identification et à l'optimisation des sources et natures des financements) et la mise sur orbite de marché (appui à la concrétisation réussie du projet). 
Quant à l'accompagnant, il vise les trois principaux facteurs clés de succès du lancement d'une start-up. Il s'agit du créateur, du projet et des perspectives du marché. Son métier consiste à traiter des problèmes particuliers, transmettre des savoirs ou des connaissances spécifiques, à stimuler et motiver l'entrepreneur en vue des performances personnelles et organisationnelles. Les logiques d'action du créateur sont tellement impactées par ses besoins d'être, de savoir et de pouvoir au point de reconstituer le substrat de son identité entrepreneuriale. Ces trois besoins constituent les composantes fondamentales de l'identité du créateur que l'accompagnant doit inscrire dans sa démarche d'expert-conseil (Murnieks, Mosakowski, 2007 ; Cardon et al., 2009; Hoang, Gimeno, 2010). Ces mêmes composantes de l'identité entrepreneuriale sous-tendent le pouvoir d'actions du créateur au sens des théories des ressources et de l'effectuation (Teece, 2007 ; Sarasvathy, 2008).

Aussi, l'objectif du présent article est de cerner le contenu des trois composantes de l'identité entrepreneuriale du créateur technologique. Notre analyse se focalise sur l'entrepreneur de la start-up. Il est surtout question de mettre en évidence les processus interactifs qui lient le créateur technologique à l'organisation qu'il a créée. Ces processus interactifs font appel à l'imbrication de plusieurs phénomènes dont les plus significatifs concernent les éléments de motivations et de compétences sous-tendant le projet initial, la socialisation dans un contexte technologique très évolutif, l'identification à une organisation avide de ressources et de compétences hautement qualifiées, les relations de partage avec des communautés financières et technologiques très exigeantes. Le décryptage de tels processus conduit à enrichir la pédagogie d'accompagnement conduite par les experts-conseils de l'entrepreneuriat technologique.

Pour parvenir à cet objectif de recherche, une étude qualitative est conduite sous forme d'entretien collectif (focus group) réunissant 8 créateurs de start-up. Les discussions ont été enregistrées et retranscrites intégralement en mars 2015. Le corpus ainsi obtenu est passé au crible des méthodes d'analyse sémantiques et structurales (Mucchielli, 1974; Ghiglione, 1978 ; Ghiglione et al., 1985). L'approche interprétativiste conduit à faire valoir l'importance des composantes identitaires au niveau de l'accompagnement du créateur d'une start-up.

Au préalable de ce résultat essentiel, un rapide éclairage de portée théorique est exposé dans la première partie de notre travail. Ceci mobilise les développements récents de la recherche managériale qui insistent sur l'exploitation des ressources clés, des systèmes de valeurs et des connaissances comme voie d'avenir dans l'appréhension des composantes de l'identité entrepreneuriale du créateur d'entreprise. La deuxième partie relève d'une démarche inductive. Celle-ci a trait aux composantes identitaires et aux logiques d'action des entrepreneurs technologiques enquêtés. Les composantes identitaires mises en évidence prennent la forme de leviers d'action pour l'expert-conseil du créateur technologique.

\section{Une mise en perspective théorique de l'identité entrepreneuriale}

\subsection{Les besoins d'être, de savoir et de pouvoir au cœur de l'identité entrepreneuriale}

L'identité de soi est le produit de plusieurs attentes individuelles. Celles-ci relèvent de détours et de retours, de refoulements et d'activations du refoulé pour constituer le besoin d'être. Sa construction se fait de manière dynamique, multidimensionnelle et complexe. Elle se transmet par des socialisations successives : histoire biographique, parcours scolaire, expériences professionnelles, valeurs et représentations collectives, etc. Ces processus de socialisation sont à la fois des déterminants de la construction de l'identité et des facteurs d'influence de cette même identité (Vibert, 2011).

En même temps, la construction progressive de l'identité contribue à orienter l'individu dans son contexte, donne du sens à ses expériences et fournit des indications pour l'action. Si l'individu choisit une orientation entrepreneuriale, il imagine les situations d'activités à cette orientation y ajuste son comportement. Ceci le fait passer d'une situation où il se conforme aux attentes des autres (du fait de ses rôles familiaux, amicaux et professionnels). Il finit par se façonner une nouvelle image de soi qui prend la forme de l'identité professionnelle. 
Cette configuration intègre plusieurs éléments et se développe graduellement à travers les synthèses et reconfigurations successives de soi. Pour Erikson (1968), l'identité est ainsi le produit (unifié, cohérent, stable) d'un processus de constitution de soi, continuellement ouvert et interactif, qui doit être validé par le sujet et reconnu par les autres. Elle est mise en intrigue en termes dynamiques par la concurrence entre une exigence de concordance et l'admission de discordances (Ricoeur, 1990). L'identité se réajuste constamment et c'est dans l'engagement au cœur de l'action et dans la relation avec l'autre au sein des interactions que le sujet se développe et construit son identité. Il agit par tâtonnement et de manière exploratoire. Les situations inhabituelles qu'il rencontre peuvent susciter la création de nouvelles étiquettes mais celles-ci doivent être justifiées, validées, ratifiées.

Dans la construction de son histoire de vie, le sujet concilie la diversité et la variabilité des événements en une interprétation homogène, fruit d'une négociation avec soi-même mais dont les termes d'accord ne sont pas réglés et fixés une fois pour toute (Strauss, 1992). Perceptible dans des gestes et faits quotidiens, l'identité du sujet peut donc être multiple, multiforme (Ruano-Borbalan, 2004), peut faire l'objet de remises en question, de relectures, de reformulations, voire d'oblitérations. La pertinence des représentations multiples au sens de l'assimilation/différenciation et de l'objectivation/appropriation est réelle (Marc, 1997 ; Tap, 1997 ; Delcourt, 1999). Il s'agit de représentations valorisantes de soi et ouvertes à l'apprentissage.

L'apprentissage valide le besoin de savoir. En fait, la motivation dans la construction de l'identité professionnelle s'effectue de façon véritable lorsqu'il existe une dynamique de structuration à partir d'éléments concrets, omniprésents dans le cadre d'un accompagnement : de nouvelles représentations, des identifications par le vécu de nouvelles expériences, l'acquisition de nouvelles compétences et l'utilisation de tous les acquis du processus d'accompagnement. Toutes les situations qui sont vécues par l'individu confortent, consciemment ou inconsciemment le processus identitaire. L'individu est soumis à des facteurs d'apprentissage endogène (sa personnalité, son système de valeurs, ses normes, etc.) et exogènes (l'entreprise, l'université, les relations avec les différents partenaires de l'entreprise), capables de le conduire à une évolution de ses acquis, de son comportement, de ses repères, de ses projets, de ses méthodes de gestion. Le besoin de pouvoir trouve alors une réponse adéquate. Autrement dit, les éléments moteurs interviennent non pas séparément dans le processus identitaire mais d'une manière interactive, mettant en mouvement un jeu d'articulations, de relations, d'évolutions, de régulations propres à l'émergence d'un système favorable à l'action professionnelle réussie. La fonction d'entrepreneur s'inscrit dans ce même système avec des compétences spécifiques.

Ainsi, la construction de l'identité entrepreneuriale exige un système d'interactions entre :

- les motivations personnelles et les missions assignées à l'organisation créée ;

- les connaissances et les compétences entrepreneuriales nécessaires ;

- les éléments facilitateurs au processus entrepreneurial et les aspirations du créateur.

Ces interactions font état de trois types de motivations entrepreneuriales au prisme des trois besoins initialement évoqués: un besoin de réalisation qui s'exprime dans le domaine professionnel, un besoin d'appartenance qui est du domaine affectif et un besoin de pouvoir, pouvoir en tant qu'acquisition des compétences, qui fait partie du domaine cognitif. Ces mêmes interactions permettent de comprendre et d'expliquer comment l'environnement (école, structures d'accompagnement, etc.) agit sur le soi (ou l'être) et comment le soi agit sur l'acquisition, la construction du savoir et du savoir-faire pour générer des comportements entrepreneuriaux (Obrecht, 2016). La connaissance de soi, les motivations et les compétences entrepreneuriales évoluent sous l'effet de l'accompagnement.

Autrement dit, tout individu effectue constamment une relecture de soi qui peut se produire à trois moments (Brown, 1994) : la taxonomie (le positionnement, le rôle par rapport à l'autre : Chef d'entreprise/ employé) ; la narration (la relation d'agence ou d'intimité entre soi et l'autre : la domination ou la coopération) ; l'imputation (l'évaluation de soi en termes de capacité de prise de responsabilité par 
rapport à l'autre). Le discours identitaire fait donc aussi le lien entre le niveau individuel (logiques égocentrées) et celui du collectif (logiques individuelles et pratiques de mise en scène de soi au sein d'un groupe), y compris dans la création d'une nouvelle entreprise (Hogg, Terry, 2000).

En définitive, la construction identitaire reflète l'histoire personnelle de chacun et constitue l'une des modalités cruciales des pratiques et des représentations individuelles. L'histoire individuelle comprend plusieurs éléments différents : l'interaction de l'individu avec ses parents, l'apprentissage des rôles liés à son sexe, l'éducation reçue dans son milieu, etc. L'acte d'entreprendre offre à l'individu la liberté de poursuivre ses propres motivations, ses rêves et ses désirs quand il crée une nouvelle entreprise. Cette activité entrepreneuriale est infusée de sens car elle est une expression de l'identité individuelle ou une conception de soi qui sert d'ascenseur pour la mobilisation des motivations, des compétences et des ressources (Kimberly, 1979 ; Murnieks, Mosakowski, 2007 ; Cardon et al., 2009 ; Hoang, Gimeno, 2010).

\subsection{Une extension vers l'approche fondée sur les ressources}

L'approche fondée sur les ressources est étendue au domaine de l'entrepreneuriat (Alvarez, Busenitz, 2001 ; Alvarez, Barney, 2002 ; Arthurs, Busenitz, 2006 ; Zhara et al., 2006). On reconnaît à l'entrepreneur des capacités personnelles spécifiques qui forment une véritable ressource critique. Il s'agit de « métacompétences » au sens de la version dynamique de la resource-based-theory de Teece (2007). Ces méta-compétences déterminent le pouvoir d'action de l'entrepreneur dans des environnements changeants. Elles prennent la dimension d'une identité entrepreneuriale ouverte et dynamique dans le modèle de l'« entrepreneur effectuel » de Sarasvathy (2008). En effet, elles se déclinent en :

- Capacités consubstantielles à la personne de l'entrepreneur : le besoin d'être de son identité (who I am ?) et ses connaissances ou le besoin de savoir (what I know ?);

- Accompagnants et autres parties prenantes (whom I know ?) avec les interdépendances entre l'entrepreneur et les acteurs en présence, interdépendances qui déterminent son pouvoir d'action ou le besoin de pouvoir (what can I do ?).

Dans le processus d'effectuation engagé par l'entrepreneur (lequel fait ses choix en fonction des effets susceptibles d'être obtenus avec tels et tels moyens et non en fonction d'un objectif prédéterminé), les parties prenantes jouent un rôle central car c'est de leur engagement que dépend la possibilité de poursuivre le processus de développement des ressources. De même, la référence à l'identité débouche sur la question des valeurs de l'individu entrepreneur, sachant que les rôles constitutifs de l'identité d'une personne ainsi que ses valeurs sont socialement déterminés et donc exposés à la diversité des sociétés et de leurs cultures.

L'identité d'un individu se construit en effet selon deux versants, celui du rapport à soi et celui du rapport à autrui, que la théorie psychologique de l'identité structurale analyse en termes de rôles. Elle résulte d'« une internalisation individuelle de rôles conformes aux attentes sociales qui portent sur des comportements rattachables à une fonction ou à des statuts » (Murnieks, Mosakowsky, 2007). L'identité de l'individu entrepreneur sera donc un construit d' « identités multiples interactives » (époux ou épouse, père ou mère, leader d'une organisation, etc.) en plus de l'identité entrepreneuriale. Mais il trouve son unité par le fait que l'individu est aussi un porteur de valeurs qui servent de principes de conduite dans la vie de toute personne : ce sont des structures mentales qui existent à un niveau d'abstraction supérieur aux attitudes. Or, selon Hitlin (2003), les valeurs se développent dans des contextes sociaux, se réfèrent par des symboles à des éléments culturels significatifs et constituent un aspect du soi qui est nécessaire et fondamental, mais non coercitif. Les valeurs, socialement calibrées, sont profondément ancrées chez l'individu et agissent, selon l'auteur cité, comme «une force de cohésion de l'identité personnelle ». Elles sont souvent fortement façonnées par la religion (Audretsch et al., 2007). Dans certains contextes 
le lien est direct entre d'une part le facteur religieux et d'autre part la propension de l'individu à devenir entrepreneur ou la structuration locale de l'action entrepreneuriale (Dana, 2010) ainsi que la construction des connaissances nécessaires. C'est une manière de rendre dynamiques les différentes composantes de l'identité entrepreneuriale comme l'indique le schéma ci-dessous.

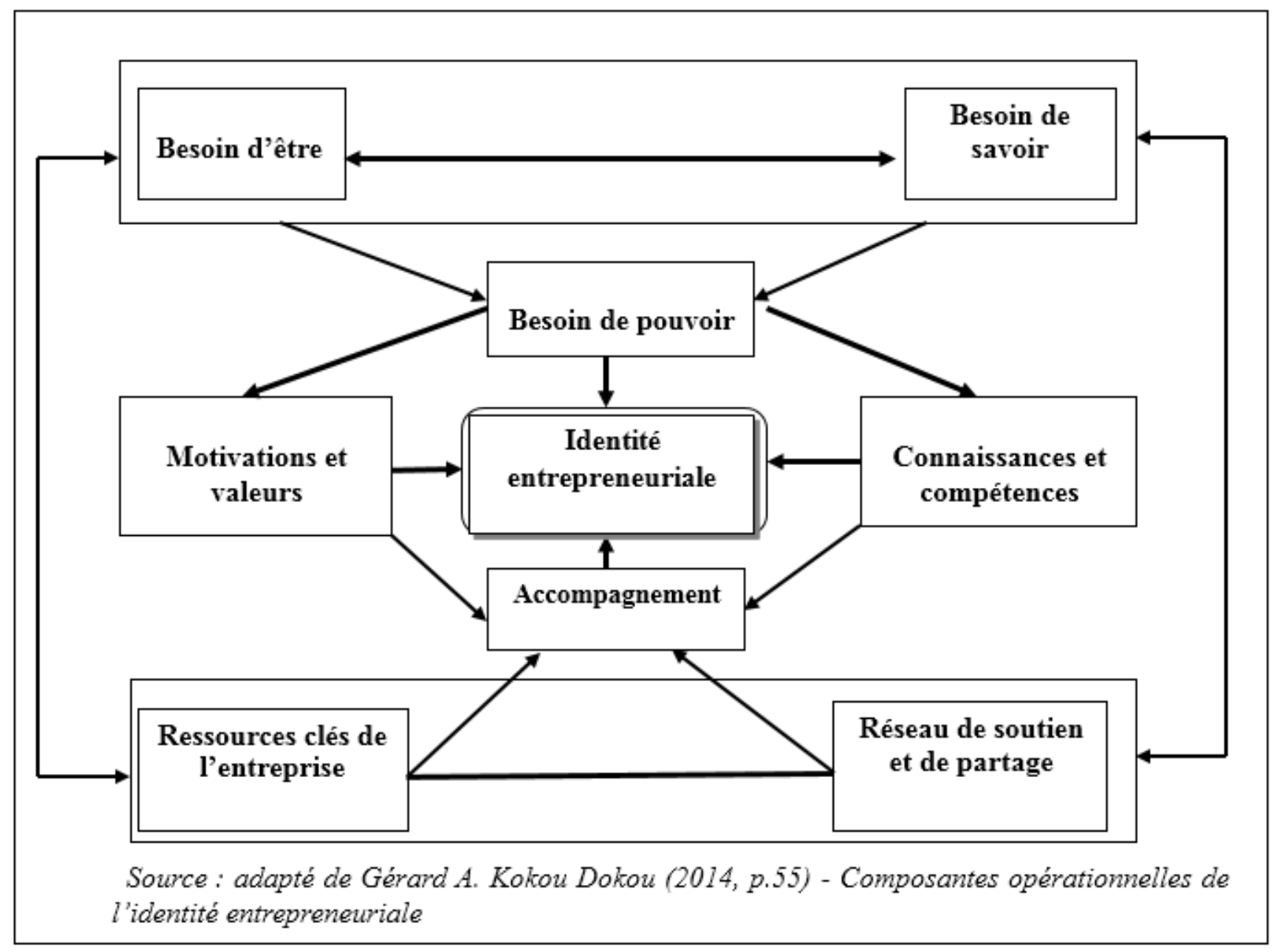

Schéma 1. Composantes opérationnelles de l'identité entrepreneuriale

Le contenu de notre figure suggère les principaux leviers opératoires qui constituent l'identité entrepreneuriale. Le processus de construction de cette identité est aidé par l'accompagnement entrepreneurial et son influence sur la création d'entreprise. L'identité entrepreneuriale est construite à travers l'appartenance du sujet à plusieurs ensembles et pourrait se définir comme une structure polymorphe, dynamique, dont les éléments constitutifs sont des aspects psychologiques et sociaux en rapport à la situation relationnelle à un moment, d'un individu comme acteur social. Les éléments pertinents, dans la situation de création d'entreprise, disparaissent au profit d'autres éléments quand la situation se modifie sous l'influence de l'accompagnement entrepreneurial. Les personnels de l'accompagnement entrepreneurial façonnent d'une façon ou d'une autre les motivations et la vision du créateur. Ils lui donnent la capacité d'agir sur sa propre définition de soi.

Par ce processus d'accompagnement, l'individu définit les comportements conscients ou inconscients et adaptés à mettre en place afin d'atteindre ses « victoires identitaires finalisées » contre un adversaire qui peut être soi-même, les autres en interaction concrète ou l'environnement social. L'une des finalités pour l'individu créateur est la reconnaissance de son existence dans le système social et économique. Ce qui implique à la fois que ce système lui reconnaisse son appartenance et une place spécifique et qu'il ressente subjectivement cette reconnaissance. Aidé par le processus d'accompagnement entrepreneurial, en affirmant son identité, le créateur affirme, par le même fait, le référentiel de son comportement, de ses méthodes de management, de ses produits, etc. 
Dans le processus entrepreneurial l'action personnelle du créateur est déterminante. Les connaissances issues des formations suivies, les expériences héritées du parcours professionnel et les valeurs familiales sont décisives. N'oublions pas que le créateur débute sa carrière entrepreneuriale avec un noyau cognitif initial structuré par un ensemble de croyances, de convictions et de comportements. Ce noyau cognitif qui oriente ses premières actions, se renouvelle et se consolide grâce au processus d'apprentissage. Il constitue une des bases essentielles de la différence managériale. Il structure le style personnel du créateur et lui signifie ce qu'il faut faire et ce qu'il ne faut pas faire. "Les traits structurels de l'environnement se présentent de la même façon pour tout le monde mais n'ont pas les mêmes conséquences pour tous. Les entrepreneurs efficaces se distinguent par leur capacité à détecter et à utiliser à leur profit les opportunités tout en se protégeant contre les difficultés » (Bouchikhi, Kimberley, 1994, p. 117).

Une telle capacité résulte de compétences plurielles renforcées par la formation continue de l'entrepreneur. Les actions de formation ont souvent une répercussion qui va au-delà de la réalisation des projets pris en compte. Elles contribuent à favoriser le développement de la culture entrepreneuriale et de l'apprentissage stratégique. La meilleure adéquation peut être cherchée entre la théorie et la pratique. Ceci consacre le caractère global de l'accompagnement qui s'appuie sur :

- Les facilitateurs et les facteurs stratégiques de risque du processus entrepreneurial.

- Les ressources clés à mobiliser pour l'entreprise en démarrage.

- Les réseaux de soutien et de partage de l'entrepreneur.

Ces trois dimensions prennent une connotation particulière dans le cas de la start-up. En effet, la start-up se définit généralement comme une jeune entreprise innovante qui a un fort potentiel de développement et qui nécessite beaucoup d'investissements dans le but de générer une croissance rapide avec un niveau de risques élevé. On la retrouve souvent dans le domaine du numérique ou les nouvelles technologies. C'est surtout un état d'esprit, une manière de voir le monde qui caractérise le mieux l'entrepreneur de la start-up. Quel que soit son secteur d'activité, la start-up porte en elle l'ambition de capter une partie de la valeur du marché et d'acquérir une position dominante sur celui-ci. En ce sens, Steve Blank et Bob Dorf (2013, p. 25) l'ont définie comme « une organisation temporaire à la recherche d'un business model industrialisable, rentable et permettant la croissance ». Et à Eric Ries de préciser que la start-up est «une institution humaine conçue pour créer un nouveau produit ou service dans des conditions d'incertitude extrême» (2011, p. 27). Les trois autres dimensions identitaires d'une telle organisation concernent les caractéristiques clés de l'entrepreneur, les composantes particulières de l'entreprise et le contexte environnemental (Littunen et al., 1998; Solmossy, 2000 ; Tödtling, Wanzenböck, 2003).

Ces trois dimensions permettent de distinguer de façon significative les entreprises technologiques qui réussissent de celles qui échouent. Un entrepreneur technologique sait combiner les ressources techniques, humaines et financières. Les ressources technologiques sont primordiales. Mais pour les exploiter, il faut des ressources humaines très qualifiées en connaissances et compétences techniques. Et les ressources financières permettent d'avoir les meilleurs collaborateurs pour pouvoir développer et exploiter les ressources technologiques.

\section{Une mise en évidence des composantes identitaires de l'entrepreneur de start-up}

\subsection{Constitution du corpus d'analyse et dispositif d'exploitation utilisé}

On entend par corpus un ensemble de textes réunis sous-tendant un objectif de recherche. Le nôtre est constitué de matériau informationnel issu d'un focus group réunissant 8 créateurs de start-up du Nord de la France. La méthode de focus group consiste à réunir de 6 à 12 personnes volontaires représentatives pour le sujet de recherche retenu. Le principe de base est respecté avec nos 8 participants. Les principaux thèmes d'échanges concernent : leurs motivations et intérêts à être entrepreneur, leurs 
valeurs et leurs missions en tant qu'entrepreneurs, leurs principales compétences et celles indispensables au métier d'entrepreneur technologique, les avantages et les inconvénients d'être entrepreneur, les facilitateurs de leur propre cheminement d'entrepreneur, leurs besoins en matière d'accompagnement, les défis et aspirations pour les 5 ans à venir, leur perception de la situation de l'entrepreneuriat en général et dans leur région en particulier. Tous les dialogues, de trois heures en moyenne, ont été enregistrés et transcrits intégralement pour constituer le corpus d'analyse.

Le corpus constitué a été analysé par le biais du système ALCESTE (Analyse des Lexèmes Cooccurrents dans un Ensemble de Segments de Textes). C'est une méthodologie complète d'analyse de données qui se définit par trois approches : approche lexicale grâce à la statistique discursive et la lexicométrie, approche d'analyse de contenu consistant à découper le texte en unités de contexte et approche d'analyse de données classifiant ces mêmes unités de contextes (Reinert, 1998). En ce sens, ALCESTE entre dans le cadre général des recherches en analyse de données linguistiques (Benzécri, 1982; Lebart et al., 2000). Elle consiste principalement en l'étude des lois de distribution du vocabulaire dans un corpus sous-tendue par les approches multidimensionnelles ou multivariées telles que l'analyse factorielle des correspondances et les classifications ascendantes hiérarchiques (C.A.H) ou descendantes hiérarchiques (C.D.H).

Le mode opératoire du système ALCESTE relève d'un processus en quatre étapes. L'étape 1 est destinée à reconnaître les discours de chacun des enquêtés à partir de plusieurs outils lexicaux. Au niveau de l'étape 2, Alceste découpe le corpus en unités de textes, appelées unités de contexte élémentaires. S'ensuivent l'analyse des vocabulaires spécifiques à ces mêmes unités et leur classification. C'est sur la base des classes identifiées que les perceptions et les logiques d'actions des enquêtés sont explicitées pour être interprétées. L'étape 3 fournit les principaux fichiers des résultats les plus significatifs à l'analyste. On y trouve les différentes classes retenues, leur dépendance mutuelle, le vocabulaire dominant tel que les présences et absences significatives des formes dans les classes, ainsi que les éléments d'identification des enquêtés. Enfin l'étape 4 conduit à l'obtention définitive de la classification. Tout ceci favorise l'analyse et l'interprétation de l'ensemble des résultats.

Les quatre étapes de l'exploitation de l'ensemble du corpus ont mis en évidence les spécificités des logiques d'actions propres aux entrepreneurs de start-up enquêtés. Ces spécificités sont fortement corrélées (test de khi2) à leurs perceptions des portées des nouvelles technologies, notamment numériques. Elles sont explicitées dans les développements qui suivent.

\subsection{Principales composantes identitaires mises en évidence}

Le graphe ci-dessous (schéma 2) traduit un des résultats clés de l'exploitation des données discursives exploitées. Il indique clairement l'importance de la technologie dans le processus entrepreneurial du créateur.

La technologie est au cœur du processus entrepreneurial du créateur enquêté. Il s'agit bien de start-up qui mobilise des moyens humains, financiers et de conseils. Les partenaires bancaires occupent une place importante. Pour conduire les actions nécessaires à la réussite de l'aventure entrepreneuriale, le conseil des spécialistes ou des experts est incontournable. Le travail en équipe et en communauté est impératif. Il permet d'ajuster et de compléter ses connaissances et compétences. D'où l'intérêt de mesurer ou d'évaluer systématiquement les connaissances et compétences en présence aujourd'hui pour pouvoir exister demain. Les contextes multiples d'aujourd'hui sont à prendre en compte de manière systémique. Il s'agit des contextes familial, professionnel, institutionnel, économique, social, culturel, national et international. Ces différents contextes sont mis au service de l'activité entrepreneuriale. La précision que fournit un des enquêtés est significatif dans ce sens : «mes aspirations et défis futurs qui doivent mobiliser des moyens financiers, c'est internet, c'est le développement international». 


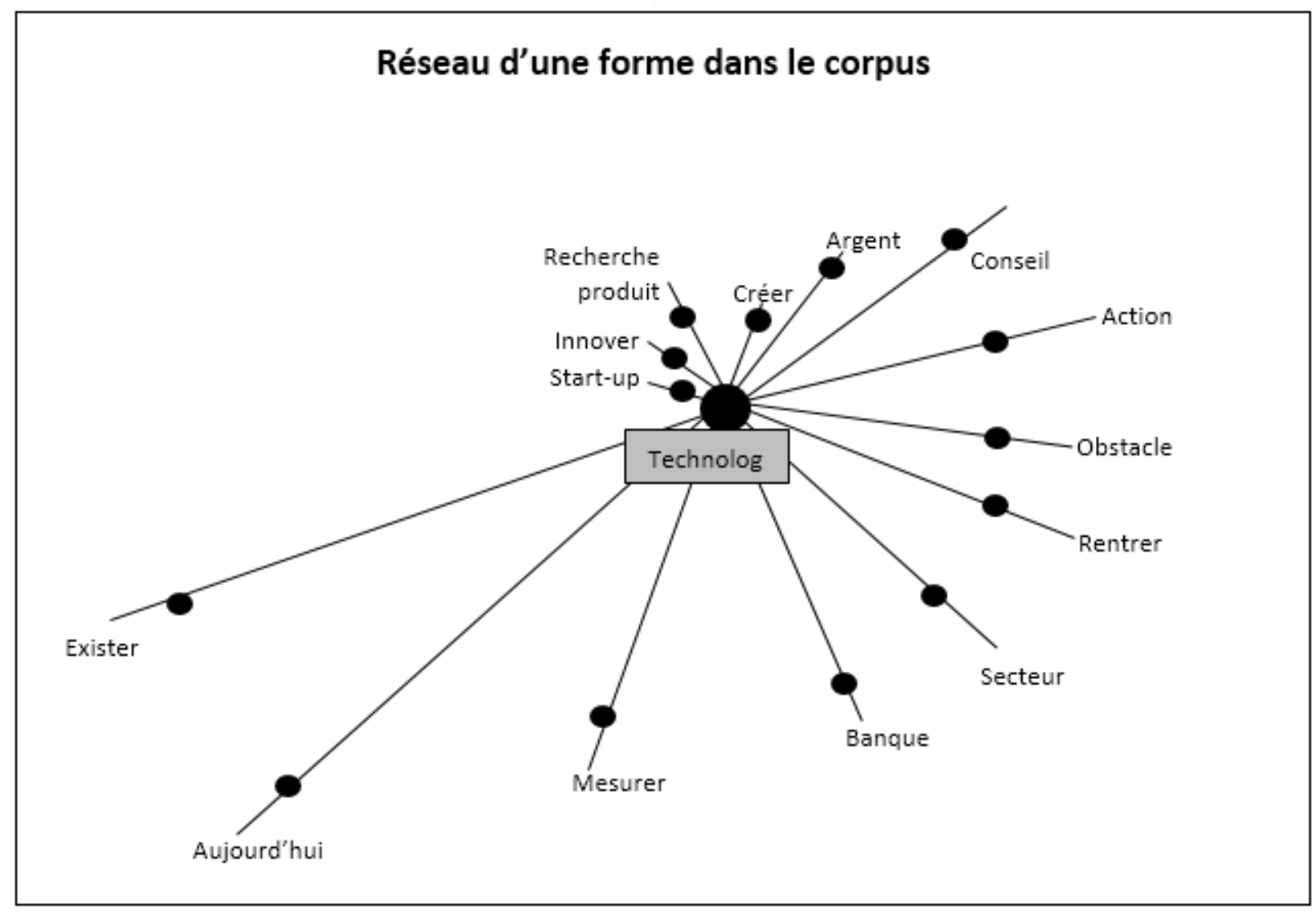

Schéma 2. Graphe de la forme lexicale significative issue du corpus étudié

Mais ceci requiert un niveau d'études plus élevé et des compétences plurielles : "aujourd'hui, il faut que tu sois un leader charismatique, un adepte des technologies nouvelles, un audacieux et un poly-compétent face à ces technologies et aux différentes activités ». Il s'agit d'acquis cognitif exprimé autrement : «dans notre génération, beaucoup ont plusieurs compétences parce que nous sommes bien formés, on nous a habitués très tôt à travailler en équipe, à travailler des projets; c'est peut-être prétentieux, mais c'est la réalité ». De telles exigences sont facilitées par l'usage des technologies numériques. C'est ce que révèlent les mots recherche, produit, innover, start-up avec un indice de Khi supérieur à $70^{1}$.

Les ressources cognitives permettent à l'entrepreneur technologique de combiner au mieux les difficultés du contexte socio-économiques à celles de son organisation. Par ailleurs, une partie du vocabulaire a une tonalité positive et se rapporte à la passion des entrepreneurs pour leur métier. Les verbatim qui suivent sont significatifs dans ce sens $(\mathrm{khi} 2=89)$ : «j'aime bien m'occuper de l'entreprise et j'aime bien mon travail de chef d'entreprise, la passion pour les technologies nouvelles en général et pour le numérique en particulier, et l'envie de créer quelque chose, de la valeur, cette passion et ces envies sont constamment là ; elles sont enfouies en moi et me guident tous les jours ». Ainsi, l'entrepreneur technologique se trouve formaté par les facteurs fondamentaux de l'évaluation de soi et ceux relatifs aux ressources émotives et cognitives que l'on retrouve dans le tableau ci-dessous.

1. Précisons que dans le système ALCESTE, le coefficient Khi2 (métrique du Khi2) permet d'apprécier la significativité du lien ou de l'association entre deux unités de contexte ou occurrences classées ou deux classes. Les couples (ou associations) les plus significatifs doivent avoir une valeur de Khi2 supérieure à 4 . 


\begin{tabular}{|c|c|}
\hline $\begin{array}{l}\text { Dimensions } \\
\text { de l'identité } \\
\text { entrepreneuriale }\end{array}$ & $\begin{array}{l}\text { Perception des entrepreneurs technologiques en termes de verbatim } \\
\text { par ordre d'importance décroissant }\end{array}$ \\
\hline $\begin{array}{l}\text { Besoin d'être : } \\
\text { motivations } \\
\text { de l'entrepreneur } \\
\text { et ressources clés } \\
\text { de l'entreprise }\end{array}$ & $\begin{array}{l}\text { - Nouvelles technologies et compétences du personnel }(\mathrm{khi} 2=89) \\
\text { - Confiance en ses capacités ou compétences en technologies numériques } \\
\text { et management stratégique }(\mathrm{khi} 2=89) \\
\text { - Passion, réalisation de soi, qualité de vie }(\mathrm{khi} 2=86) \\
\text { - Réactivité face à la mondialisation }(\mathrm{khi} 2=85) \\
\text { - Indépendance }(\mathrm{khi} 2=81) \text {, autonomie }(\mathrm{khi} 2=79) \text {, pouvoir }(\mathrm{khi} 2=69) \\
\text { - Ressources et sécurité financières }(\mathrm{khi} 2=69) \\
\text { - Equilibre sport, culture et travail }(\mathrm{khi} 2=69) \\
\text { - Adaptabilité/flexibilité }(\mathrm{khi} 2=69) \\
\text { - Recrutement des compétences face à la peur de manquer son coup } \\
\text { et aux expériences limitées }(\mathrm{khi} 2=55)\end{array}$ \\
\hline $\begin{array}{l}\text { Besoin de savoir : } \\
\text { connaissances, } \\
\text { réseau de soutien } \\
\text { et de partage }\end{array}$ & $\begin{array}{l}\text { - Ouverture au progrès technologique, à l'innovation, à la créativité, } \\
\text { à l'international }(\mathrm{khi} 2=89) \\
\text { - Ouverture aux nouvelles pratiques et cultures }(\mathrm{khi} 2=87) \\
\text { - Esprit d'équipe }(\mathrm{khi} 2=87) \\
\text { - Soutien familial }(\mathrm{khi} 2=87) \\
\text { - Niveau de formation }(\mathrm{khi} 2=85) \\
\text { - Recherche du soutien financier et moral }(\mathrm{khi} 2=68)\end{array}$ \\
\hline $\begin{array}{l}\text { Besoin de pouvoir } \\
\text { Principales } \\
\text { compétences }\end{array}$ & $\begin{array}{l}\text { - Stratégie }(\mathrm{khi} 2=92) \\
\text { - Défi internet, croissance / ouverture internationale }(\mathrm{khi} 2=89) \\
\text { - Travail en équipe }(\mathrm{khi} 2=89) \\
\text { - Peur de l'échec ou de ne pas être à la hauteur des attentes }(\mathrm{khi} 2=85) \\
\text { - Réactivité permanente pour la fidélisation et le développement } \\
\text { de la clientèle }(\mathrm{khi} 2=85) \\
\text { - Obligation de résultats }(\mathrm{khi} 2=84) \\
\text { - Conception de nouveaux projets }(\mathrm{khi} 2=81) \\
\text { - Délégation }(\mathrm{khi} 2=70) \\
\text { - Télétravail et innovations technologiques }(\mathrm{khi} 2=69) \\
\text { - Expériences des accompagnants, conseillers et des collaborateurs }(\mathrm{khi} 2=69) \\
\text { - Etablissement des priorités }(\mathrm{khi} 2=68) \\
\text { - Nombreuses responsabilités }(\mathrm{khi} 2=45) \\
\text { - Stress et incertitude }(\mathrm{khi} 2=35) \\
\text { - Longues heures de travail }(\mathrm{khi} 2=30)\end{array}$ \\
\hline
\end{tabular}

Tableau. Principales occurrences les plus significatives au sens de Khi2

L'analyse interprétative du contenu de ce tableau s'articule autour des trois composantes identitaires initialement retenues.

\section{Motivations entrepreneuriales en cohérence avec les compétences technologies}

Plusieurs motifs alimentent le désir de démarrer, d'acheter ou de reprendre une entreprise. Les motivations identifiées par les entrepreneurs y sont de deux natures : l'exploitation passionnées des nouvelles technologies et la recherche d'indépendance. "Ma principale motivation est de travailler avec passion, avec les nouvelles technologies, avec des gens que j'ai choisis sur des sujets que j'ai choisis ». Les traits motivationnels exprimés sont plus nombreux. Il s'agit de la réalisation d'un rêve, la recherche de la qualité de vie, l'indépendance, le pouvoir et l'autonomie. L'entrepreneur de start-up est également poussé par le but de prouver ses capacités et ses compétences par l'exploitation des nouvelles technologies. Celles-ci constituent les ressources clés de son organisation. 
Dans la cadre de cette étude, les créateurs de start-up mettent l'accent sur certaines valeurs :

- Ouverture à l'innovation, à la créativité, à l'international, au changement, à la compétition ;

- Recherche de nouveaux savoirs, nouvelles cultures et pratiques.

Ces mêmes valeurs suscitent en eux le désir de changer le monde, d'être utiles à la société, d'être libres et indépendants. Un répondant précise : «je veux faire coïncider mes valeurs personnelles avec celles qui prévalent dans mon travail de chef d'entreprise, c'est dans ce sens que les technologies numériques m'aident énormément ».

Par ailleurs, il apparait que l'innovation est perçue comme une nécessité dans des champs concurrentiels souvent mondialisés et affectés par les changements technologiques. La maîtrise de ces changements est facilitée par le niveau d'études, le type de formation et une espèce de dépassement de soi qui est une véritable source de bonheur et de plaisir. Plaisir d'œuvrer pour le progrès, bonheur du travail bien fait, reviennent constamment et résident dans la conciliation travail-famille et valeurs personnelles/valeurs professionnelles.

\section{Besoin de pouvoir centré sur les compétences technologiques et stratégiques}

Le besoin de pouvoir s'appuie sur les compétences technologiques et la capacité à travailler en équipe. Mais une analyse plus fine du corpus informationnel exploité permet de constater que les compétences « stratégiques » sont prises au sérieux. Celles-ci sont associées aux dimensions opérationnelles de « délégation et partage des tâches » constamment abordées pendant les 3 heures du focus group. D'autre part, on remarque qu'il s'agit également de compétences projectives qui s'appuient sur les technologies numériques, la créativité, la formation académique, le fait de savoir s'entourer des bonnes personnes et d'être bien conseillé ou accompagné, la flexibilité, la recherche de nouveauté et du soutien familial des points de vue moral et financier.

Le créateur technologique a suivi un cheminement d'entrepreneur grâce à la confiance dans ses capacités, au soutien moral et financier de la part de ses proches et des banquiers, aux conseils des accompagnants et à ses collaborateurs. L'appui de l'entourage immédiat, des experts-conseils et des communautés financières et technologiques apparaît incontestable. Un tel appui semble consolider les composantes de l'identité entrepreneuriale du créateur technologique.

\subsection{Trois principales composantes identitaires à prendre en compte dans l'accompagnement de l'entrepreneur technologique}

\section{Des caractéristiques clés : motivations, connaissances et expériences}

Les résultats de notre étude montrent que le créateur technologique a une forte orientation entrepreneuriale. Celle-ci se traduit par une propension à agir de manière autonome, une volonté d'innover, de prendre des risques et une tendance à être agressif vis-à-vis des opportunités du marché (Lumpkin, Dess, 1996). Même si l'innovation reste la base de toute création d'entreprise, celle d'une start-up est fondée sur les connaissances mobilisées requérant un haut niveau de qualification et des compétences techniques particulières (Nerlinger, 1998 ; Seeger, 1997).

Au niveau de nos enquêtés, ces variables sont le fruit de leur histoire de vie, des formations universitaires suivies et de l'accès à l'information facilitée par l'ouverture aux technologies numériques (Darmon, 2010). En la matière, ils éprouvent tous un réel désir d'amener à la pratique un ensemble de techniques qu'il maîtrise (Shane, 2004). Ils ont également la volonté de prouver qu'un concept technologique vaut la peine d'être exploité (Gasse, 2002). Leurs motivations et ambitions sont sous-tendues par de solides croyances en leur projet. Dans les faits, de telles croyances sont relayées par de nombreux impératifs. Ils se sont pliés à l'entraînement à la création, à l'élaboration d'un plan d'affaires avec l'étude de faisabilité 
technique et financière du projet, l'identification de contacts pertinents, l'élaboration d'une première liste de clients potentiels, etc. (Schutjens, Wever, 2000 ; Dahlqvist et al., 2000).

Il va de soi que la création d'une entreprise fondée sur l'innovation et la connaissance demande une activité de préparation plus étendue et de meilleure qualité que celle qui est nécessaire pour les entreprises non innovantes. Par ailleurs, le processus de création est plus long pour les entreprises technologiques qu'il ne l'est pour les entreprises classiques (Allen, Stearns, 2003). L'étendue du niveau de connaissances peut impacter favorablement ce processus.

Une des principales différences entre les entrepreneurs technologiques et les entrepreneurs classiques concernent le niveau de scolarité. En effet, les créateurs d'entreprise technologique sont plus éduqués que ceux des créateurs classiques (Borges et al., 2003). Avec 36 ans de moyenne d'âge, nos 8 entrepreneurs technologiques sont très éduqués $(\mathrm{bac}+5)$ mais très peu expérimentés professionnellement (Christensen et al., 2001 ; Colombo, Delmastro, 2001). Une expérience professionnelle significative contribue au succès de l'entreprise en démarrage (Wiklund, Shepherd, 2001). C'est particulièrement vrai quand il y a une similarité entre la nouvelle entreprise et l'organisation dans laquelle l'entrepreneur a travaillé préalablement (Brüderl et al., 1996; Cooper et al., 1994). En tous les cas, la majorité des entrepreneurs technologiques ont acquis ou développé leurs connaissances techniques et managériales en travaillant préalablement dans des organisations, dans les mêmes secteurs d'activités que ceux dans lesquels ils créent leur entreprise par la suite (Bernasconi, 2000 ; Kourilsky, Walstad, 2002). Seuls 2 sur les 8 s'inscrivent dans ce cadre. Deux autres sont issus de l'essaimage des PME familiales du même secteur d'activité (Lasch, Le Roy, Yami, 2005). L'essaimage technologique est un processus par lequel une nouvelle entreprise se crée sur la base d'un ou de plusieurs éléments transférés d'une organisation parente ou incubatrice. Mais tous s'appuient sur une équipe entrepreneuriale de deux à cinq membres (Kadji-Youaleu, Filion, 2002).

La personnalité de l'entrepreneur joue aussi un rôle important dans la mesure où il a une grande influence sur son organisation. Au sein de l'équipe entrepreneuriale, il se met dans une position d'apprentissage continu ; ceci favorise la mise en place d'une autre logique de structuration de l'avenir. Ses perceptions évoluent plus rapidement. Il reste ouvert à toutes innovations afin de ne pas pénaliser son entreprise et de ne pas frustrer son équipe. Il finit par créer un système de gestion responsabilisant un maximum de personnes et peut engager le remodelage de l'organisation de l'entreprise pour qu'elle puisse être la plus compétitive possible. On est en présence de compétences et de ressources collectives et évolutives considérées comme un des principaux facteurs de leur succès (Aspelund et al., 2005; Colombo, Grilli, 2005 ; Filion et al., 2003).

\section{La nature des ressources propres à l'entreprise technologique}

Les 8 entrepreneurs enquêtés ont fait valoir, dans leur cheminement, l'importance de plusieurs ressources accessibles. L'importance des ressources accessibles pour une entreprise technologique est déterminante pour son développement et ses performances. Les faits montrent que l'insuffisance de ressources propres est une des causes de la disparition des jeunes entreprises (Zhao, Aram, 1995). Un des objectifs fondamentaux d'une entreprise jeune est d'évoluer d'une organisation pauvre en ressources à une organisation riche en ressources. Les moyens qui lui font défaut sont de natures variées et se rapportent entre autres au capital, à la technologique, aux compétences managériales et humaines.

Les ressources technologiques constituent l'ingrédient primordial pour le développement des nouveaux produits et services (Shane, 2001). Le degré d'innovation de la technologie exploitée est un des facteurs de succès pour les nouvelles entreprises technologiques (Aspelund et al., 2005). Cependant, les ressources technologiques évoluent dans un degré de complexité plus élevé que pour les entreprises classiques. Ces évolutions requièrent des efforts en $R \& D$ supplémentaires et des compétences techniques incontournables. Les entrepreneurs technologiques, en raison de leur formation et de leurs expériences, sont préparés pour initier et coordonner les ressources technologiques qui leur sont nécessaires. Toutefois, plusieurs 
travaux soulignent qu'il manque à ces entrepreneurs d'autres compétences très importantes pour assurer le succès de la nouvelle entreprise : compétences en marketing et vente, en gestion, en finance et en comptabilité (Albert, 2000 ; Gartner et al., 1997). Cette faiblesse est prise en compte par nos 8 enquêtés. Ils sont sensibles à tous les aspects du fonctionnement de leur entreprise : la vision stratégique, le mode de gouvernance ou le management, le niveau des performances, les méthodes de travail, la commercialisation, la gestion des ressources humaines, la logistique, etc.

Ils sont également assistés d'un associé ou d'une équipe entrepreneuriale fondatrice (Ruef et al., 2003; Schutjens et Wever, 2000; Teal et Hofer, 2003). Grâce à leurs experts-conseils, ils tiennent également compte des autres besoins. Il s'agit du personnel qualifié, d'équipements et de locaux adaptés au temps nécessaire au développement d'une nouvelle technologie et au financement des technologies complémentaires. D'ailleurs, les exigences du personnel qualifié les conduisent à adopter des stratégies spéciales de recrutement et de fidélisation (Foo, Tan, 2002). Certains entrepreneurs n'hésitent pas à proposer aux salariés le statut d'associés. L'équipe entrepreneuriale prend ainsi la forme d'un véritable groupe pluridisciplinaire en termes d'expérience et de connaissances (Aspelund et al., 2005 ; Lasch et al., 2005).

Il apparaît que le coût élevé de la technologie de base exploitée et des ressources humaines qualifiées, la complexité du marché prospecté et la durée du processus de développement des nouvelles technologies, rendent les besoins en ressources financières de l'entrepreneur technologique plus élevées que ceux de l'entrepreneur classique (Albert, 2000 ; Gasiglia et al., 2000). Malheureusement, peu d'entrepreneurs ont accès au capital de risque ou à d'autres sources de financement conséquent (Heirman, Clarysse, 2004 ; Gorman et al., 2005 ; Shepherd, Zacharakis, 2001). Il va sans dire que 1'accès aux ressources financières est l'une des principales difficultés rencontrées par les entrepreneurs lors de la création d'une entreprise technologique (Auken, 2001 ; Kourilsky, Walstad, 2002 ; Shepherd, Zacharakis, 2001).

Dans tous les cas, le mode de gestion le plus courant s'appuie sur des logiques de proximité (spatiale, hiérarchique, fonctionnelle, temporelle, voire familiale) avec des entités encastrantes. L'équilibre entre vie privée et vie professionnelle et les conseils des professionnels d'accompagnement (en matière d'activation et de construction des compétences entrepreneuriales les plus adaptées) renforce ces logiques de proximités. C'est ce que Obrecht (2016) traduit en termes de proximalités tant la pédagogie d'accompagnement impacte l'identité entrepreneuriale du créateur à partir de son parcours, ses traits de personnalité et de ses aptitudes personnelles. L'esprit d'entreprise qui se manifeste par de solides convictions et par l'affectif symbolique et irrationnel est naturellement concerné. La connaissance de soi, les motivations et les compétences entrepreneuriales évoluent sous l'effet de l'accompagnement dans une perspective de confiance partagée.

\section{Un environnement centré sur la confiance partagée dans des réseaux opérationnels et stratégiques}

Les 8 entrepreneurs technologiques enquêtés font valoir des particularités saillantes: une solide culture des nouvelles technologies, l'accès aux études supérieures, la recherche de soutien moral et de financement, les besoins de conseil et d'accompagnement. La recherche d'une réelle osmose entre l'identité d'entrepreneur (en phase avec les nouvelles technologies) et celle de l'entreprise créée est également permanente (Gasse, Tremblay, 2014). Ils tentent de maintenir une telle osmose grâce à l'ouverture aux connaissances et pratiques nouvelles (Trope, Liberman, 2010). Ils sont conscients de ne pouvoir échapper à l'influence positive ou négative des communautés dont ils dépendent (Stam, 2015). En fait, «le démarrage d'une entreprise ou d'une innovation est un processus collectif qui commence dans l'environnement de l'entreprise et qui est soutenu par la confiance partagée dans des réseaux de proximité, stimulés par les acteurs régionaux »(Julien, 2003, p. 175). Ils ont des besoins spécifiques en interaction particulière avec l'économie locale (Sapienza et al., 2003). C'est le cas des activités de recherche et développement, des connaissances et d'expertises particulières. L'accès et l'exploitation efficace de telles ressources permettent de mieux gérer l'intensité concurrentielle très forte au niveau du marché mondial (Nowak, Grantham, 2000). 
C'est dire que l'entrepreneur technologique a besoin du soutien institutionnel à l'échelle régionale et locale (Feld, 2012). Le soutien institutionnel peut même encourager des regroupements intersectoriels à l'échelle régionale ou nationale (Diamantopoulos, 2011 ; Pezzini, 2012; 2013). C'est une manière d'élargir et de consolider les collaborations qui se nouent avec une multitude d'acteurs : des partenaires financiers, technologiques et commerciaux. On sait désormais que la mise en œuvre de tels partenariats conditionne l'émergence, la survie et la performance de nouvelles organisations technologiques (Nicolaou, Birley, 2003; Shane, Stuart, 2002). Désormais, c'est tout un écosystème qui s'anime autour de l'entrepreneur technologique pour l'accompagner. La relation entre d'une part, le réseau personnel de l'entrepreneur et d'autre part, le démarrage et la croissance de l'entreprise créée a été clairement démontrée tant la connexion à divers réseaux est bien le principal critère distinctif discriminant les projets d'innovation qui réussissent par rapport à ceux qui défaillissent.

L'analyse de Granovetter (1983 et 1985) concernant les réseaux à signaux faibles et à signaux forts prend tout son sens. Les premiers, mal connus et mal compris de l'entrepreneur, font émerger l'information originelle à partir de laquelle naît par la suite l'innovation. Ils permettent de découvrir des potentialités d'affaires. Les seconds, avec lesquels la communication est aisée et empreinte d'une confiance mutuelle entre ses composantes, joue davantage un rôle de validation des informations reçues par ailleurs, et de soutien dans le processus d'innovation. Le rôle du partenariat de confiance a également été souligné par Fourcade (1994) qui fait remarquer que le réseau est primordial pour des petites entreprises qui ambitionnent d'acquérir les technologies de pointe et de bénéficier d'un effet de taille. C'est le cas des cercles d'amis, des clubs sportifs ou encore des liens familiaux, qui peuvent participer à la formation de la réputation d'un entrepreneur (Bernasconi, Monsted, 2000).

Les réseaux facilitent les relations d'échanges de ressources et de compétences, ouvrent de nouvelles opportunités de marché et renforcent les actions de remodelage de l'environnement au profit de l'entreprise (Zimmerman, Zeitz, 2002). Ces relations d'échanges sont facilitées par les incubateurs technologiques (Dambron, 2008 ; Menu, 2011 ; Matray, Poisat, 2014 ; Dubeux, 2014). D'ailleurs, de nombreux incubateurs technologiques s'associent aux activités concernant les innovations de transformation sociale (Lévesque et al., 2014 ; Bouchard et al., 2015). Ce faisant, ils cherchent à être de véritables accélérateurs de jeunes entreprises aux concepts novateurs de tous genres. L'appui accélérateur s'effectue au travers des sources de financement plus accessibles, des logistiques d'hébergement ou encore des espaces de coworking, d'appui à l'internationalisation, etc.

C'est dans ce système de gestion partenariale que s'inscrit le club des entrepreneurs accompagnés. L'accompagnement post-création se veut ainsi à la fois individuel et collectif. Le choix de la démarche partenariale vient de la volonté de l'entrepreneur qui cherche à transformer constamment son organisation. A côté des pressions de l'environnement, l'action intentionnelle du dirigeant est un des principaux moteurs de la transformation organisationnelle. La coopération devient ainsi un outil de gestion des facteurs de rigidité et d'inertie (invariants culturels bloquants) organisationnelles. Progressivement, elle conduit l'entreprise à éviter d'être une entité passive ou simplement réactive face aux stimuli de l'environnement.

\section{Conclusion}

La première étape de notre article souligne l'importance de l'identité entrepreneuriale des créateurs technologiques souvent encastrés dans une culture de flexibilité, d'ouverture d'esprit, d'innovation et de réactivité. La prise en compte de ce besoin d'être identitaire fonde la pertinence scientifique de l'objet de cette contribution : améliorer l'accompagnement du processus de création des entreprises technologiques. Les composantes identitaires restituées (de façon très synoptique) par notre enquête sont significatives dans ce sens. Ces composantes identitaires sont à la base des logiques entrepreneuriales mises en œuvre par les créateurs technologiques. Ces mêmes composantes identitaires prennent la forme des besoins d'être, de savoir et de pouvoir et expriment des différenciations de sens dont la captation conduit à des approches mobilisant les ressources de tous genres au profit du développement de 
l'entrepreneuriat technologique (World Economic Forum, 2013): l'accessibilité aux marchés, le capital humain et la main d'œuvre, l'investissement et la finance, un système de soutien en conseil ou mentorat, les aides au démarrage, l'éducation et la formation, le soutien moral et culturel.

Du point de vue individuel, nous avons plus ou moins déterminé les facteurs clés de succès pour un entrepreneur technologique. Nous avons vu par exemple que l'entrepreneur avec son expérience a un impact relatif et significatif sur la réussite de son projet. Cependant un entrepreneur ne doit pas compter seulement sur son expérience mais surtout aussi sur ses ressources technologiques. D'ailleurs un entrepreneur technologique est une personne capable d'accéder et de coordonner les ressources technologiques, humaines et financières en vue d'une meilleure profitabilité (Stam, 2014).

Cependant, il est nécessaire d'approfondir ces résultats empiriques ainsi que la démonstration proposée au travers des arguments épistémologiques, théoriques et pratiques. Le décryptage de l'accompagnement des entrepreneurs technologiques et développeurs s'inscrit dans cette optique. Cet approfondissement devra mettre l'accent sur d'autres composantes identitaires pouvant sous-tendre la pédagogie d'accompagnement entrepreneurial en écho aux similitudes, différenciations et interconnections des générations de créateurs/repreneurs d'entreprise technologique. Les trois zones de construction interactive de l'identité entrepreneuriale seront clarifiées de manière opérationnelle : la zone de prédisposition à mobiliser les connaissances et les compétences (individu et besoin d'être), la zone d'appropriation ou d'acquisition et de construction des connaissances et des compétences (besoin de savoir) et la zone de réalisation ou de mise en œuvre active et/ou proactive des compétences dans un projet technologique finalisé par l'individu (besoin de pouvoir-faire).

\section{Références}

ALBERT P., La création d'entreprises de haute technologie, Histoire d'entreprendre- Les réalités de l'entrepreneuriat, Éditions EMS, Paris, p. 149-160, 2000.

Allen K., Stearns T., Nascent High Tech Entrepreneurs: The Who, Where, When and Why, in G. D. Libecap (ed.), Advances in the Study of Entrepreneurship, Innovation and Economic Growth, vol. 14, London, p. 195-218, 2003.

ALlen K., SteARns T., Technology Entrepreneurs, in W. B. Gartner, K. G. Shaver, P. D. Reynolds (eds), Handbook of entrepreneurial dynamics - The process of business creation, Sage, London, p. 438-448, 2004.

ALVAREZ S.A., BUSENITZ L.W., The Entrepreneurship of Resource-Based Theory, Journal of Management, vol. 27, p. 755-775, 2001 .

Alvarez S.A., BARNEY J.B., Resource-Based Theory and the Entrepreneurial Firm, Hitt M.A., Ireland R.D., Camp S.M., Sexton D. L., (eds), Strategic Entrepreneurship: Creating a New Mindset, Oxford, Blackwell Publishers, p. 89-105, 2002.

ARThuRS J.D., BusENITZ L.W., Dynamic Capabilities and Venture Performance: The Effects of Venture Capitalists, Journal of Business Venturing, vol. 21, n 2, p. 195-215, 2006.

ASPELUND A., BERG-UtBy T., SKJEVDAL R., Initial Resources, Influences on New Venture Survival: A Longitudinal Study of New Technology-Based Firms, Technovation, vol. 25, p. 1337-1347, 2005.

AUDRETSCH D.B., BÖNTE W., TAMVADA J.P., Religion and Entrepreneurship, Social Science Research Network (en ligne), 2007.

AUKEN H., Financing Small Technology-Based Companies: The Relationship between Familiarity with Capital and Ability to Price and Negotiate Investment, Journal of Small Business Management, vol. 39, p. 240-258, 2001.

BERNASCONI M., Les modèles de développement des entreprises high tech, in M. Bernasconi, M. Monsted (dir.). Les start-up high tech : Création et développement des entreprises technologiques, Dunod, Paris, p. 59-75, 2000.

Bernasconi M., Monsted M., Les start-up high tech, Dunod, Paris, 2000.

BENZECRI J.-P., Histoire et préhistoire de l'analyse des données, Dunod, Paris, 1982.

BIRCH D., Small Business : Slump? What Slump ?, interview dans Fortune, Decembre 2002.

BLANK S., DORL B., Le Manuel du créateur de start-up : Etape par étape, bâtissez une entreprise formidable, Les Editions Diateino, Paris, 2013. 
BORGES C., BERNASCONI M., FILION L.-J., La création des entreprises de hautes technologies - examen de la documentation, Cahier de recherche 2003-11, Chaire d'entrepreneuriat ROGERS J.-A., - BOMBARDIER., HEC Montréal, 2003.

Bouchard M., Trudellec J., Briand L., Klein J.L., Levesque B., Longtin D., Pelletier M., A Relational Database to Understand Social Innovation and its Impact on Social Transformatin, NichOLlS A., SiMON J., GABRIEL M., (eds), New Frontiers in Social Innovation Research, Palgrave MacMillan, London, p. 69-85, 2015.

BOUCHIKHI H., KimBERLEY J., Entrepreneurs et Gestionnaires, Ed. d’Organisation, Paris, 1994.

BRown D.D., Discursive Moments of Identification, Current Perspectives in Social Theory, vol. 14, p. 269-292, 1994.

CARDon M.S., Wincent J., The Nature and Experience of Entrepreneurial Passion, Academy of Management Review, vol. $34, \mathrm{n}^{\circ} 3$, p. 511-532, 2009.

CAStrogiovanni G., Pre-Start-Up Planning and the Survival of New Small Businesses: Theoretical Linkages, Journal of Management, vol. 22, $\mathrm{n}^{\circ}$ 6, p. 801-822, 1996.

COLOMBo M., GRILLI L., Founders' Human Capital and the Growth of New Technology-Based Firms: A Competence-Based View, Research Policy, vol. 34, p. 795-816, 2005.

Colombo M., Delmastro M., Technology-Based Entrepreneurs: Does Internet Make a Difference?, Small Business Economics, vol. 16, p. 177-190, 2001.

CoOper A.C., Gimeno-Gascon F.J., Woo C.Y., Initial Human and Financial Capital as Predictors of New Venture Performance, Journal of Business Venturing, vol. 9, nº 5, p. 371-395, 1994.

DAHLQVIST J., DAVIDSSON P., WIKLUND J., Initial Conditions as Predictors of New Venture Performance: A Replication and Extension of the Cooper et al. Study, Enterprise \& Innovation Management Studies, vol. 1, n 1, p. 1-17, 2000.

DAMBRON P., Les clusters en France, pourquoi les pôles de compétitivité ?, L’Harmattan, Paris, 2008.

DANA L.P., Entrepreneurship and Religion, Edward Elgar, Cheltenham, 2010.

DARMON M., La socialisation: Domaines et approches, Armand Colin, Paris, 2010.

Diamantopoulos M., Cooperative Development Gap in Québec and Saskatchewan 1980 to 2010: A Tale of Two Movements, Canadian Journal of Nonprofit and Social Economy Research / Revue canadienne de recherche sur les OBSL et l'économie sociale, vol. 2, n 2, p. 6-24, 2011.

DокоU KокоU G.A., Identité territoriale, proximités locales et capacités entrepreneuriales, in G. A. Kokou Dokou (dir.), Acteurs territoriaux, identités et capacités entrepreneuriales, L'Harmattan, Paris, 2014.

ERIKSON E.H., Identity Youth and Crisis, New York, W. W. Norton, 1968.

Eastman J., Iyer R., LiaO-Troth S., Williams D., Griffin M., The Role of Involvement on Millennials' Mobile Technology Behaviors: The Moderating Impact of Status Consumption, Innovation, and Opinion Leadership, Journal of Marketing Theory and Practice, vol. 22, n 4, p. 455-470, 2014.

FOURCADE C., Localisation versus globalisation : les stratégies résiliaires des petites entreprises, communication à la 39ème ICSB World Conference, Strasbourg, 1994.

FELD B., Start-Up Communities: Building an Entrepreneurial Ecosystem in your City, John Wiley \& Sons Inc, New York, 2012.

Foo, M.D., TAN H.H., Critical Incidents in High-Tech Start-Ups: A Singapore Study, in Phan P. (ed.), Technological entrepreneurship, p. 185-204, 2002.

Gartner W., Starr J., Bhat S., Predicting New Venture Survival: An Analysis of Anatomy of a Start-up, Cases from Inc, Magazine, Journal of Business Venturing, vol. 14, n 2, p. 215-232, 1997

Gasiglia C., GueYe S., PISTRE N., La valorisation, enjeu central de la logique financière des start-up, in M. Bernasconi, M. Monsted (dir.), Les start-up high tech : Création et développement des entreprises technologiques, Dunod, Paris, $\mathrm{p}$. 167-179, 2000.

GASSE Y., Les entrepreneurs technologiques: le profil des chercheurs-entrepreneurs, miméo, 2002.

GASSE Y., TREMBlay M., Etude sur les entrepreneurs et les repreneurs québécois de générations $X$ et $Y$, FSA, Université Laval, Québec, Canada, 2014.

Ghiglione R., Matalon B., Les enquêtes sociologiques, théories et pratiques, Armand Colin, Paris, 1978.

GHiglione R., MATALON B., BACRi N., Les dires analysés, l'analyse propositionnelle du discours, PUF, Paris, 1985. 
GORMAN G., Rosa P.J., FASERUCK A., Institutional Lending to Knowledge-Based Business, Journal of Business Venturing, vol. 20, p. 793-819, 2005.

GranovetTER M., The Strength of Weak Ties: Network Theory Revisited, Sociological Theory, vol. 1, p. 201-233, 1983.

GranovetTer M., Economic Action and Social Structure: The Problem of Embeddedness, American Journal of Sociology, vol. $91, \mathrm{n}^{\circ} 3$, p. 481-510, 1985.

Heirman A., Clarysse B., How and Why do Research-Based Start-Ups Differ at Founding? A Resource-Based Configurational Perspective, Journal of Technology Transfer, vol. 29, p. 247-268, 2004.

Hitlin S., Values at the Core of Personal Identity: Drawing Links Between Two Theories of Self, Social Psychology Quarterly, vol. 66, n², p. 118-137, 2003.

HoAng H., Gimeno J., Becoming a Founder: How Founder Role Identity Affects Entrepreneurial Transitions and Persistence in Founding, Journal of Business Venturing, vol. 25, $\mathrm{n}^{\circ}$ 1, p. 41-53, 2010.

Hogg M.A., TerRY, D.J., Social Identity and Self-Categorization Processes in Organizational Contexts, Academy of Management Review, vol. 25, p. 121-140, 2000.

JULIEN P.-A., Innovation et entrepreneuriat, in P. Mustar, H. Penan H. (dir.), Encyclopédie de l'innovation, Economica, Paris, p. 167-178, 2003.

KADJI-Youaleu C., FiLION L.-J., Essaimage technologique : Examen de la documentation, Cahier de recherche 2002-14, Chaire d'entrepreneurship Maclean Hunter, HEC Montréal, 2002.

KIMBERLY J., Issues in the Creation of Organizations: Initiation, Innovation and Institutionalization, Academy of Management Journal, 22, 437-457, 1979.

KOURILSKY M.L., WALSTAD W.B., The Early Environment and Schooling Experiences of High-Technology Entrepreneurs: Insights for Entrepreneurship Education, International Journal of Entrepreneurship Education, vol. 1, p. 87-106, 2002.

LASCH F., LE ROY F., YAMI S., Les déterminants de la survie, de la croissance des start-up TIC, Revue française de gestion, vol. 31, n 155, p. 37-56, 2005.

Lebart L., Morineau A., Piron M., Statistique Exploratoire Multidimensionnelle, Dunod, Paris, 2000.

LitTUnen H., Storhammar E., NenONEn T., The Survival of Firms over the Critical First 3 Years and the Local Environment, Entrepreneurship and Regional Development, vol. 10, p. 189-202, 1998.

LUMPKIN G.T., DeSS G.G., Clarifying the Entrepreneurial Orientation Construct and Linking It to Performance, Academy of Management Review, vol. 21, n 1, p. 135-172, 1996.

MARC E., L’identité personnelle, Sciences Humaines, Hors-Série : Identité, Identités, vol. 15, p. 6-8, 1997.

MATRAY M., PoISAT J., Les PTCE, une dynamique citoyenne et institutionnelle d'innovations sociales, Cahiers du CIRTES Hors-Série 4, Presses Universitaires Louvain, Louvain, 2014.

MENU S., Les Pôles de compétitivité, un nouveau pilotage de la politique industrielle ? Bilan en Ile-de-France, Politiques et management public, vol. 28, $\mathrm{n}^{\circ} 1,2011$.

MuCCHIELli R., L'analyse de contenu, E.M.E., Paris, 1974.

MURNIEKS C.Y., MOSAKOWSKI E.M., Who am I? Looking Inside the Entrepreneurial Identity, in Frontiers of Entrepreneurship Research Proceedings, Babson College Entrepreneurship Research Conference (BCERC), Madrid, Spain, vol. 7-9, 2007.

NERLINGER E., Standorte und Entwicklung junger innovativer Unternehmen: Empirische Ergebnisse für West-Deutschland, Baden-Baden, Schriftenreihe des Zentrum für Europäische Wirtschaftsforschung (ZEW), vol. 27, n 3, p. 111-132, 1998.

NiCOLAOU N., BIRLEY S., Academic Networks in a Trichotomous Categorisation of University Spinouts, Journal of Business Venturing, vol. 18, n 3, p. 333-359, 2003.

NowaK M.J., GRANTHAM C.E., The Virtual Incubator: Managing Human Capital in the Software Industry, Research Policy, vol. 29, p. 125-134, 2000.

OBRECHT J.-J., Sustainable Entrepreneurship Education: A New Field for Research in Step with the 'Effectual Entrepreneur', International Journal of Entrepreneurship \& Small Business (en cours de publication), 2016

PEZZINI E., La capacité d'innovation et d'exploration des nouveaux secteurs coopératifs. Le cas italien, in M.-J. Brassard, E. Molina (dir.), L'étonnant pouvoir des coopératives, Québec, Sommet international des coopératives, p. 571-582, 2012.

PeZzINI E., Les associations des coopératives. Potentialités, défis, modèles d'organisations, Conférence internationale sur la recherche en économie sociale du CIRIEC International, Anvers, p. 24-26 octobre, 2013. 
REINERT M., Processus catégorique et co-construction des sujets dans différents récits, Linguistique et Psychanalyse, Colloque de Cerisy-La-Salle, septembre, 1998.

Reynolds P., BygRAVE W., AUtio E., HAY M., GEM 2002 Summary Report, Global Entrepreneuship Monitor, Washington, 2002.

RIES E., The Lean Start-Up: How Today's Entrepreneurs Use Continuous Innovation to Create Radically Successful Businesses, New York, Crown Publishing Group, 2011.

RICOEUR P., Soi-même comme un autre, Le Seuil, Paris, 1990.

RUANO-BORBALAN J.-C., L'identité, une ressource pour l'action, in C. Halpern, Ruano-Borbalan, J.-C., Identité(s). L'individu. Le groupe. La société, Editions Sciences Humaines, Paris, 2004.

RUEF M., Aldrich H.E., CARTER N.M., Homophily, Strong Ties, And Isolation among U.S. Entrepreneurs, American Sociological Review, vol. 68, p. 195-222, 2003.

SAPIENZA H.J., AUtio E., ZAHRA S., Effects of Internationalization on Young Firms' Prospects for Survival and Growth, Academy of Management Best Conference Paper, 2003.

Schutjens V., WeVer E., Determinants of New Firm Success, Papers of Regional Science, 79, 135-159, 2000.

SARASVATHY S.D., Effectuation: Elements of Entrepreneurial Expertise, Edward Elgar, Cheltenham, 2008.

SEEGER H., Ex-Post Bewertung der Technologie und Gründerzentren durch die erfolgreich ausgezogenen Unternehmen und Analyse der einzel undregionalwirtschaftlichen Effekte, Münster, Hannoversche Geographische Arbeiten, vol. 53, $\mathrm{n}^{\circ} 4$, p. 1-15, 1997.

Shane S., StUART T., Organizational Endowments and the Performance of University Start-Ups, Management Science, vol. 48, n 1, p. 154-170, 2002.

SHANE S., Encouraging University Entrepreneurship? The Effect of the Baby-Dole Act on University Patenting in the United States, Journal of Business Venturing, vol. 19, p. 127-151, 2004.

SHANE S., Technological Opportunities and New Firm Creation, Management Science, vol. 47, n 2, p. 205-220, 2001.

SHANE S., Technology Regimes and New Firm Formation, Management Science, vol. 47, n 9, p. 1173-1190, 2001.

SHEPHERD D.A., ZACHARAKIS A., The Venture Capitalist-Entrepreneur Relationship: Control, Trust and Confidence in Cooperative Behaviour, Venture Capital: An International Journal of Entrepreneurial Finance, vol. 3, p. 129-149, 2001.

Solmossy E., Entrepreneurial Dimensions: The Relationship of Individual, Venture and Environmental Factors to Success, Entrepreneurship, Theory \& Practice, p. 79-80, 2000.

Stam E., Entrepreneurial Ecosystems and Regional Policy: A Sympathetic Critique, Ultrecht School of Economics, Koopmans Research Institute, p. 15-37, 2014.

STRAuSs A., Miroirs et masques, Métailié, Paris, 1992.

TAP P., Marquer sa différence, Sciences Humaines, Hors-Série : Identité, Identités, vol. 15, p. 9-10, 1997.

Teal E.J., Hofer C.W., New Venture Success: Strategy, Industry Structure, and the Founding Entrepreneurial Team, The Journal of Private Equity, Fall, p. 38-51, 2003.

TEECE D.J., Explicating Dynamic Capabilities: The Nature and Microfoundations of (Sustainable) Enterprise Performance, Strategic Management Journal, vol. 28, p. 1319-1350, 2007.

Trope Y., LiBerman N., Construal-Level Theory of Psychological Distance, Psychological Review, vol. 117, $\mathrm{n}^{\circ} 2$, online, 2010

VIBERT P., L'identité : Un nouveau chantier théorique ?, in O. Lazzarotti, P.-J. Olagnier, L'identité. Entre Ineffable et effroyable, Armand Colin, Paris, 2011.

WIKLUND J., SHEPHERD C., The Sustainability of the Entrepreneurial Orientation-Performance Relationship, Entrepreneurship Theory \& Practice, Fall, p. 37-48, 2001.

World Economic Forum, Entrepreneurial Ecosystems Around the Globe and Company Growth Dynamics, Report Summary for the Annual Meeting of the New Champions 2013, Davos, 2013.

ZHAO L., ARAM J.D., Networking and Growth of Young Technology-Intensive Ventures in China, Journal of Business Venturing, vol. 10, $\mathrm{n}^{\circ}$ 5, p. 349-370, 1995.

Zimmerman M.A., ZeITZ G.Z., Beyond Survival: Achieving New Venture Growth by Building Legitimacy, Academy of Management Review, vol. 27, n 3, p. 414-431, 2002.

(c) 2016 ISTE OpenScience - Published by ISTE Ltd. London, UK - openscience.fr 